\title{
Psychological Problems and Intervention Strategies of Front- Line Nurses Fighting against Covid-19
}

\author{
Bo Zhou', Gan Wang ${ }^{2}$ and Yun Liü ${ }^{3 *}$ \\ ${ }^{1}$ International School of Nursing, Huangshan Vocational Technical College, Huangshan, China \\ ${ }^{2}$ Department of Psychology, Jiangsu Province Hospital of TCM, China \\ ${ }^{3}$ Department of Nursing, Nanjing University School of Medicine, China
}

\begin{abstract}
The front-line nurses who fight against the Covid-19 epidemic situation have high work intensity, high risk of infection and high risk of mental health. Psychological intervention is very important for the physical and mental health of nursing staff. Through the analysis of the working state, stress disorder and mental health factors of front-line nursing staff, this paper puts forward some psychological intervention countermeasures and suggestions, such as early psychological crisis intervention, strengthening the emergency drill of public health events, establishing professional belief, strengthening the labor protection of female nursing staff, etc., for the reference of professionals and relevant departments.
\end{abstract}

Keywords

Covid-19, Nursing staff, Mental health risk, Psychological intervention

As of September 12, who released the epidemic data of all regions around the world, with 287968 new confirmed cases and 5783 new deaths worldwide. The most serious cases in the Americas region reached 14566710 cases (119030 new cases), 505397 cases died (3463 cases), and the number of confirmed cases is still on the rise. Nurses play a key role in coping with various human health challenges [1]. In the front line of the fight against Covid-19, nursing staff is the largest new force in the rescue team [2]. Who director general Tan Desai posted a video "nursing mother" through social media, praising nurses that health workers are under the greatest pressure in the fight against the Covid-19 epidemic. This touching video shows the love and power of nurses to save lives. Their dedication and physical and mental health problems have attracted the attention of all sectors of society. This paper intends to analyze and elaborate from the perspective of psychological health of nursing staff, so as to provide certain basis for the maintenance of physical and mental health of nurses fighting against Covid-19.

The Harmfulness of Epidemic Situation and the Working State of Nursing Staff in The Process of Treatment

\section{Severity and social harmfulness of Covid-19 epi- demic}

On January 30, the World Health Organization announced that the epidemic situation of Covid-19 was listed as a public health emergency of international concern. This means that the severity and unusual nature of the outbreak has attracted international attention; the public health risk is beyond the scope of one country and requires immediate international action. Covid-19 epidemic is sudden, urgent, serious, highly uncertain and harmful to society, which causes mass anxiety and panic [3-5].

\section{Working status of front-line nurses in Covid-19 treatment}

In the Covid-19 treatment process, nursing work is very important, nursing staff are facing great nursing pressure. In isolation wards, nursing staff often take several responsibilities, not only to undertake the treatment of patients, but also to care for the daily life of patients. They also take care of the food and toilet of some seriously ill and elderly patients $[6,7]$. The work of nursing staff is divided into morning shift, afternoon shift and night shift every day, with 24-hour "three shifts". Especially when nursing critical patients, they need

*Corresponding author: Yun Liu, Department of Nursing, Nanjing University School of Medicine, 22 Hankou Road, Nanjing, Jiangsu Province 210002, China

Received: September 21, 2020

Accepted: October 09, 2020

Published online: October 11, 2020

Citation: Zhou B, Wang G, Liu Y (2020) Psychological Problems and Intervention Strategies of Front-Line Nurses Fighting against Covid-19. J Nurs Pract 3(1):165-168 
long-term continuous observation and monitoring of disease changes. They have long working hours and heavy workload, and need high concentration and emotional input. Because of the shortage of protective clothing and inconvenient wearing and taking off, some nursing staff dare not drink water, do not go to the toilet, or can only use disposable diapers for 4 hours or even 8 hours. Especially, they cannot change sanitary napkins in time during physiological period, which brings great challenges to the physiology and psychology of these young women, and also increases the chance of reproductive tract infection. In order to prevent family infection, most of the first-line rescue workers stay in hotels and cannot go home to reunite with their families for a month or more. Especially in the intensive care unit, the nursing staff have high risk and difficult conditions. It is a common phenomenon that the patients need to turn over and take care of them regularly, accompany them for a long time, and lack of rest and sleep [8].

\section{Covid-19 Treatment of Psychological Stress and Post-Traumatic Stress Disorder in Front- Line Nurses}

\section{Psychological stress and psychological stressors}

2019 Novel coronavirus (2019-nCoV) and severe acute respiratory syndrome coronavirus (SARS-CoV), Middle East respiratory syndrome coronavirus (Middle East respiratory syndrome syndrome, Middle (OV) is highly similar and more infectious [9-11], which brings great psychological pressure to front-line rescue workers, who are worried that they, their families and colleagues will be infected. In mid July, the World Health Organization (WHO) had received more than 1.4 million cases of Covid-19 infection among medical staff, which accounted for almost $10 \%$ of the global infection (at that time, more than 13.8 million cases were diagnosed). Since then, the number of infections among medical staff has continued to increase. On September 1, the Centers for Disease Control and Prevention (CDC) released a research report that from April 3 to June 19, 2020, 3248 clinical medical staff were tested in 13 state medical centers, and $6 \%$ of the respondents were finally positive for new coronavirus antibody. CDC believes that this indicates that front-line medical staff are at high risk of Covid-19 and/or transmission. The front-line nursing staff witnessed that a batch of infected persons or colleagues were sent to the ward, rescued or died. They were under great psychological pressure. In addition, they suffered from excessive fatigue caused by long-time overload continuous work, discomfort and interpersonal distance caused by wearing protective equipment and excessive disinfection, as well as the full screen of negative information on the Internet. Ross j [12] thinks: The psychological stress of Covid-19 frontline medical staff (nursing staff) is mainly manifested in physical and mental fatigue caused by long-term overwork, feeling pressure, helplessness and fear in the face of a large number of patients, worry and guilt about the illness of themselves and their families; uncertainty, depression and exhaustion about when the end is, and sadness and helplessness after seeing colleagues infected [4]. Wu [13] research found that the main sources of work pressure of nursing staff in fever clinic are: Less nurses, frequent shifts, high work intensity, no guarantee of rest and diet; insufficient protection, worrying about the safety of themselves and their families in the face of being infected by their colleagues; most patients in isolation wards show impatience, complaint, anger, fear, and even aggression. Therefore, the nurses are the direct victims of these bad emotions and behaviors, and this kind of strong stress causes the nurses' bad emotions such as tension, depression, anxiety and sadness.

\section{Long term psychological stress can increase the risk of mental and behavioral abnormalities}

Studies have shown that long-term stress also affects diet and sleep, and some nursing staff have mental symptoms such as low mood and out of control, loss of confidence in life and work [14]. The "point in" of stress disorder is characterized by depression, learned helplessness, avoidance, irritability, loss of self-confidence, while "pointing out" is characterized by increased irritability, irritability, quarrel, aggression and other mental symptoms and aggressive behaviors. Long time post-traumatic stress disorder can affect work efficiency, reduce social function, and seriously affect the physical and mental health of nursing staff. Therefore, timely and effective psychological crisis intervention is very necessary.

\section{Psychosomatic disease risk of front-line nurses treated by Covid-19}

Severe and persistent stress and emotional response can cause mental and behavioral problems, accompanied by neuroendocrine and immune system functions.

The changes of the body's physiological function and even a variety of diseases, affecting physical and mental health. Post-traumatic stress disorder caused by public health emergencies is closely related to the mental health status of nursing staff. The survey shows that the positive rate of mental health problems of employees who encounter negative events (33.7\%) is significantly higher than that of female employees (11.4\%) [15]. Long term stress disorder can cause serious physiological problems, such as chronic fatigue, insomnia, headache, muscle soreness, gastric ulcer and other psychosomatic diseases. According to a retrospective survey of professionals who undertake emergency rescue tasks in the health system, $14.7 \%$ of the rescue workers developed psychosomatic diseases after the rescue work, including skin allergy, massive alopecia, frequent cough, endocrine disorders, chronic pharyngitis, oral mucinous ulcer, chronic headache, etc. Gastric and duodenal ulcer or chronic enteritis, low back pain and so on [16]. The incidence of psychosomatic diseases of rescue workers is related to the severity, complexity and risk of the incident. The more serious the emergency is, the higher the incidence of psychosomatic diseases of emergency rescue personnel is, the more serious the condition may be.

Countermeasures and Suggestions on Psychological Intervention During and after Epidemic Situation

Timely and effective mental health promotion and psychological crisis intervention 
Timely and effective mental health promotion and psychological crisis intervention are the necessary means to avoid the occurrence of severe post-traumatic stress disorder in the future, and to ensure their physical and mental health and the quality of nursing work [17]. The intervention measures in the epidemic situation should start from many aspects, including reducing the continuous working time, increasing the communication with the outside world, establishing social support, providing psychological hotline or online consultation, encouraging nursing staff to actively respond, providing delicious food to ensure their nutritional intake, and providing appropriate support for their families. For the introverted and poor expression of nursing staff should focus on, to provide them with the opportunity of emotional disclosure and emotional vent, emotional disclosure can make mental health and physical health improved at the same time. The psychological crisis of front-line nursing staff may occur during the implementation of treatment task or after the end of treatment task. Therefore, psychological intervention should be continued after the end of the epidemic, follow-up, care and help them regain their life confidence and adapt to the new life style. At the same time, nursing staff should master the common psychological intervention techniques, such as self relaxation training, mindfulness meditation, exercise therapy, etc.

\section{Strengthen the emergency drill and training of public health emergencies}

It is suggested that attention should be paid to the participation of nursing staff in the emergency drill of public health emergencies, so as to improve the nursing work ability, emergency handling ability, emotion control ability and self-protection ability of nursing staff, and at the same time, improve the "pre-disaster preparation ability, disaster handling ability, post disaster recovery ability and feedback improvement ability" [18]. We can try relaxation training and stress vaccination training to reduce anxiety, improve individual behavior under stress situation, help relieve grief, and alleviate post-traumatic stress disorder in future life.

\section{Establish professional belief, understand and re- spect the profession of nursing staff}

At present, the educational background and personality characteristics of nursing staff are different, and the working mode, working attitude, work motivation and technical proficiency are also different, which is reflected in the quality of nursing service Patients, their families and all sectors of society should give full trust, understanding, tolerance and respect to nursing staff, and put an end to the language and behavior of contempt, humiliation and injury to nursing staff.

\section{Rational view of the physiological characteristics of women, strengthen the work of female nurs- ing staff}

The majority of the front-line nursing staff are women. The physiological structure and physiological function of female nursing staff have their particularity, which should be treated rationally. The nurses in the Covid-19 epidemic isolation ward are unable to contact with the outside world. The unit should provide them with some necessary supplies for their physiological period as much as possible to avoid embarrassment and discomfort during the physiological period. The risk of nursing staff participating in treatment during pregnancy is high, which should be eliminated from the system. During the outbreak of SARS in 2003, 12 pregnant women were infected with SARS-CoV, $57 \%$ of them had miscarriage in the first three months of pregnancy, and four of the five pregnant women in the second trimester of pregnancy gave birth prematurely; in 2014, 11 pregnant women were infected with MERS-CoV, and 10 (91\%) had adverse pregnancy outcomes; and 2019-nCov had similar pathogenic potential with SARS-CoV and MERSCoV [19]. Therefore, pregnant women should be avoided from high-risk work such as first-line rescue, and special care and protection should be given.

\section{Funding}

This study was supported by grants from Anhui University Teaching Quality Engineering Project "Huangshan Changren Hospital Demonstration Training Center of Huangshan Vocational and Technical College" (2019xqsxzx40); Anhui High Level Vocational Nursing Major (2018ylzy107); Natural Science Research Project of Anhui Universities (No. KJ2018A0955, KJ2018A0954), Establishment of quality engineering project at Institute level (No. 2020jxyj03).

\section{References}

1. Sharma SK, Nuttall C, Kalyani V, et al. (2020) Clinical nursing care guidance for management of patient with COVID-19. J Pak Med Assoc 70: S118-S123.

2. Bagnasco A, Zanini M, Hayter M, et al. (2020) COVID 19-A message from Italy to the global nursing community. J Adv Nurs 76: 2212-2214.

3. Goyal P, Choi JJ, Pinheiro LC, et al. (2020) Clinical characteristics of Covid-19 in New York city. N Engl J Med 382: 2372-2374.

4. Khalili M, Karamouzian M, Nasiri N, et al. (2020) Epidemiological characteristics of COVID-19: A systematic review and meta-analysis. Epidemiol Infect 148: e130.

5. Pung R, Chiew CJ, Young BE, et al. (2020) Investigation of three clusters of COVID-19 in Singapore: Implications for surveillance and response measures. Lancet 395: 1039-1046.

6. Davidson PM, Szanton SL (2020) Nursing homes and COVID-19: We can and should do better. J Clin Nurs 29: 2758-2759.

7. Green J, Doyle C, Hayes S, et al. (2020) COVID-19 and district and community nursing. Br J Community Nurs 25: 213.

8. Turale S, Meechamnan C, Kunaviktikul W (2020) Challenging times: Ethics, nursing and the COVID-19 pandemic. Int Nurs Rev 67: 164-167.

9. Franquet $\mathrm{T}$, Jeong $\mathrm{YJ}$, Lam $\mathrm{H}$, et al. (2020) Imaging findings in coronavirus infections: SARS-CoV, MERS-CoV, and SARS-CoV-2. Br J Radio 93: 20200515.

10. Singh A, Singh RS, Sarma P, et al. (2020) A comprehensive review of animal models for Coronaviruses: SARS-CoV-2, SARS-CoV, and MERS-CoV. Virol Sin 35: 290-304.

11. Rabaan AA, Al-Ahmed SH, Haque S, et al. (2020) SARS-CoV-2, SARS-CoV, and MERS-COV: A comparative overview. Infez Med 28: $174-184$. 
12. Ross J (2020) The exacerbation of burnout during COVID-19: A major concern for nurse safety. J Perianesth Nurs 35: 439-440.

13. Wu D, Jiang C, He C, et al. (2020) Stressors of nurses in psychiatric hospitals during the COVID-19 outbreak. Psychiatry Res 288: 112956.

14. Rana W, Mukhtar S, Mukhtar S (2020) Mental health of medical workers in Pakistan during the pandemic COVID-19 outbreak. Asian J Psychiatr 51: 102080.

15. Lien L, Haavet OR, Thoresen M, et al. (2007) Mental health problems, negative life events, perceived pressure and the frequency of acute infections among adolescents. Results from a cross-sectional, multicultural, population-based study. Acta Paediatr 96: 301-306.
16. Morren M, Dirkzwager AJ, Kessels FJ, et al. (2007) The influence of a disaster on the health of rescue workers: A longitudinal study. CMAJ 176: 1279-1283.

17. Luo YH, Li H, Plummer V, et al. (2019) An evaluation of a positive psychological intervention to reduce burnout among nurses. Arch Psychiatr Nurs 33: 186-191.

18. Jakeway CC, La Rosa G, Cary A, et al. (2008) The role of public health nurses in emergency preparedness and response: A position paper of the Association of State and Territorial Directors of Nursing. Public Health Nurs 25: 353-361.

19. Favre G, Pomar L, Musso D, et al. (2020) 2019-nCoV epidemic: What about pregnancies? Lancet 395: e40. 